\title{
CAMBIOS EN EL PERFIL FUNCIONAL DE PACIENTES CON PATOLOGÍA PSIQUIÁTRICA RESISTENTE SOMETIDOS A NEUROCIRUGÍA FUNCIONAL ESTEREOTÁCTICA
}

\author{
Lucía A. Ledesma-Torres ${ }^{1 *}$, Pamela García-Saldivar², Cristina Rodríguez-Hernández ${ }^{1}$, Omar Medrano-Espinosa ${ }^{1}$, \\ Óscar Meneses-Luna ${ }^{1}$, Francisco J. Valencia- Granados ${ }^{1}$, Manuel Hernández-Salazar ${ }^{1}$. \\ ${ }^{1}$ División de Neurociencias, Centro Médico Nacional "20 de Noviembre” ISSSTE \\ ${ }^{2}$ Facultad de Estudios Superiores Zaragoza, Universidad Nacional Autónoma de México - UNAM
}

Recibido, abril 25/2014

Concepto de evaluación, mayo 12/2014

Aceptado, mayo 28/2014

Referencia: Ledesma-Torres, L., García-Saldivar, P., Rodríguez-Hernández, C., Medrano-Espinosa, O., MenesesLuna, O., Valencia- Granados, F., Hernández- Salazar, M. (2014). Cambios en el perfil funcional de pacientes con patología psiquiátrica resistente sometidos a neurocirugía funcional estereotáctica. Acta Colombiana de Psicología, 17 (2), pp. 53-59. DOI:10.14718/ACP.2014.17.2.6

Resumen

Con el objetivo de evaluar los resultados de la Neurocirugía Funcional Estereotáctica (NFE) sobre el perfil funcional de los pacientes diagnosticados con Patología Psiquiátrica Resistente (PPR), se realizó un diseño pre/post tratamiento. 13 pacientes fueron evaluados ( $\overline{\mathrm{x}}=31 \pm 8$ años): siete mujeres diagnosticadas con algún Trastorno de la Conducta Alimentaria Resistente (TCAR); tres mujeres diagnosticadas con Trastornos Resistentes Obsesivo Compulsivo y Depresivo (TOC y DR) y tres pacientes (dos mujeres y un hombre) diagnosticados con Depresión Resistente (DR). A estos pacientes se les aplicó la Escala de funcionamiento del paciente Eje K, la cual consta de siete subescalas, y una Escala de Evaluación de la Actividad Global (EAG) antes del tratamiento y después de éste (seis meses posteriores a la NFE). Todos los grupos diagnósticos y el total de los pacientes evaluados presentaron cambios clínicos positivos; sin embargo, sólo el grupo de TCAR mostró importantes cambios clínicos de mejoría en el perfil de funcionamiento, siendo esta diferencia estadísticamente significativa (pre $M d=56$; post $M d=84 ; \mathrm{z}=-2.36, \mathrm{p}<0.05)$. Con la evidencia obtenida se observó que la NFE representa una opción terapéutica emergente efectiva orientada a disminuir el sufrimiento de los pacientes con PPR, así como a mejorar el nivel de funcionamiento global y por ende su calidad de vida. Todos los pacientes del estudio presentaban una condición de resistencia a tratamientos convencionales indicados, la cual había sido documentada por un equipo interdisciplinario experto.

Palabras clave: Patología Psiquiátrica Resistente, Neurocirugía Funcional Estereotáctica, Trastorno de la Conducta Alimentaria Resistente, Trastorno Obsesivo Compulsivo Resistente, Depresión Resistente.

\section{CHANGES IN THE FUNCTIONAL PROFILE OF PATIENTS WITH RESISTANT PSYCHIATRIC DISORDERS SUBJECTED TO STEREOTACTIC FUNCTIONAL NEUROSURGERY}

\begin{abstract}
In order to evaluate the results of the Stereotactic Functional Neurosurgery (SFN) on the functional profile of patients diagnosed with Resistant Psychiatric Pathology (RPP), a pre / post treatment design was performed. Thirteen patients were assessed ( $\overline{\mathrm{x}}=31 \pm 8$ years): seven women diagnosed with some type of Resistant Eating Disorder (RED); three women diagnosed with Resistant Obsessive Compulsive Disorder and Resistant Depression (TOC and RD), and three patients (two women and one man) diagnosed with Resistant Depression (RD). The Patient's Functioning Scale, axis K, consisting of seven subscales, and the Assessment of Global Activity Scale (AGAS) were applied before and after treatment (6 months after FSN). All diagnostic groups and all the patients assessed showed positive clinical changes. However, only the RED group showed important clinical improvement in the performance profile with a statistically significant difference (pre $\mathrm{Md}=56, \mathrm{Md}=84$ post, $\mathrm{z}=-2.36, \mathrm{p}<0.05$ ). With the evidence obtained it was noted that SFN represents an effective therapeutic option aimed at reducing the suffering of patients with RPP and improve their overall level of functioning and therefore their quality of life. All patients in the study had a condition indicating resistance to conventional treatments which had been documented by an expert multidisciplinary team.

Key words: Resistant Psychiatric Pathology, Stereotactic Functional Neurosurgery, Resistant Eating Disorder, Resistant Obsessive Compulsive Disorder, Resistant Depression.
\end{abstract}

* Félix Cuevas 540, Edif. "B” 9 p piso, Col. Del Valle, Benito Juárez, 03229 Ciudad de México, Distrito Federal, México. Tel. 01555200 5003 Ext.86880.dra.lucia.ledesma@gmail.com 


\title{
MUDANÇAS NO PERFIL FUNCIONAL DE PACIENTES CON PATOLOGIA PSIQUIÁTRICA RESISTENTE SUBMETIDOS A NEUROCIRURGIA FUNCIONAL ESTEREOTÁXICA
}

\begin{abstract}
Resumo
Con o objetivo de avaliar os resultados da Neurocirurgía Funcional Estereotáxica (NFE) sobre o perfil funcional dos pacientes diagnosticados com Patologia Psiquiátrica Resistente (PPR), realizou-se um desenho pré/pós tratamento. 13 pacientes foram avaliados $(\overline{\mathrm{x}}=31 \pm 8$ anos): sete mulheres diagnosticadas com algum Transtorno da Conduta Alimentar Resistente (TCAR); três mulheres diagnosticadas com Transtornos Resistentes Obsessivo Compulsivo e Depressivo (TOC e DR) e três pacientes (duas mulheres e um homem) diagnosticados com Depressão Resistente (DR). A estes pacientes foi aplicada a Escala de funcionamento do paciente Eixo K, a qual consta de sete sub-escalas, e uma Escala de Avaliação da Atividade Global (EAG) antes do tratamento e depois dele (seis meses posteriores à NFE). Todos os grupos diagnósticos e o total dos pacientes avaliados apresentaram mudanças clínicas positivas; porém, só o grupo de TCAR mostrou importantes mudanças clínicas de melhoria no perfil de funcionamento, sendo esta diferença estatisticamente significativa (pré $M d=56$; pós $M d=84 ; \mathrm{z}=-2.36$, $\mathrm{p}<0.05$ ). Com a evidência obtida observou-se que a NFE representa uma opção terapêutica emergente efetiva orientada a diminuir o sofrimento dos pacientes com PPR, bem como a melhorar o nível de funcionamento global e portanto sua qualidade de vida. Todos os pacientes do estudo apresentavam uma condição de resistência a tratamentos convencionais indicados, a qual havia sido documentada por uma equipe interdisciplinar de especialistas.

Palavras chave: Patologia Psiquiátrica Resistente, Neurocirurgia Funcional Estereotáxica, Transtorno da Conduta Alimentar Resistente, Transtorno Obsessivo Compulsivo Resistente, Depressão Resistente.
\end{abstract}

\section{INTRODUCCIÓN}

Los trastornos psiquiátricos, en general, suelen tratarse mediante diferentes estrategias terapéuticas convencionales tales como psicoterapia, farmacoterapia y en algunos casos terapia electroconvulsiva, o bien, la combinación de éstas. Regularmente, estos procedimientos suelen ser suficientes para la disminución de la sintomatología incapacitante. Desafortunadamente, existen casos en los que no se encuentra respuesta eficaz a pesar de diversos intentos terapéuticos convencionales e indicados para cada patología.

Son estos casos, llamados resistentes, cuya sintomatología incapacitante no disminuye con el uso de tratamientos médicos tradicionales, ni con diferentes enfoques psicoterapéuticos e incluso con terapias físicas como la electroconvulsiva o la estimulación magnética transcraneal, los que se convierten en crónicos con el paso de los años (Wang, Chang, Geng, Wang \& Gao, 2013; Luigjes, et al., 2013). Para que la patología psiquiátrica se considere como resistente se destacan diversos aspectos a tener en cuenta durante el proceso de evaluación, los cuales se refieren a la pobre o nula o respuesta clínica a todas las tentativas terapéuticas en sus diferentes modalidades y periodos de aplicación, a pesar de que éstas hayan sido bien llevadas. Este problema de la resistencia al tratamiento se repite en todo el espectro de los trastornos psiquiátricos generando enormes costos emocionales, económicos y sociales (Carrillo, Jiménez, Soto, Velasco \& Castro, 2006).

El padecimiento resistente al tratamiento tiene que ser considerado de extrema gravedad, tanto por su persistencia como por la intensidad de sus síntomas y su cronicidad, al haber tenido por lo menos una duración de cinco años, causando discapacidad o disfuncionalidad del paciente en sus actividades diarias (Oquendo, 2003).

Thase (2003) describe la patología psiquiátrica como resistente, cuando mediante la revisión del historial clínico del paciente se confirma que no ha respondido adecuadamente a todas las terapias convencionales después de un periodo mínimo de cinco años. También es indispensable descartar cualquier etiología orgánica de su trastorno mental a través un estudio sistémico, y cuantificar la gravedad de la enfermedad con las conocidas escalas de investigación neuropsiquiátrica, e integrarse tanto al estudio psicológico completo como al resto de los exámenes efectuados.

Describiéndola de diversas maneras, y aunque aún no existe un consenso internacional definitivo, Oquendo (2003) considera la patología psiquiátrica como resistente en términos de cronicidad, refiriéndose al tiempo de desarrollo de la patología; de discapacidad, en cuanto a la afectación de la patología en la vida cotidiana del paciente; de gravedad, haciendo referencia a la intensidad de la misma; así mismo, alude a la falta de respuesta a los tratamientos convencionales, principalmente farmacológicos, físicos y/o psicoterapéuticos.

Es por ello que en la actualidad, la neurocirugía funcional estereotáctica (NFE) ha representado una opción terapéutica para estos casos específicos, que se han reportado cada vez con mayor frecuencia en la literatura. Para el caso de TCAR, Wang et al. (2013) reportaron las evaluaciones pre y postratamiento, a través de estimulación cerebral profunda 
(ECP) del núcleo accumbens (NAcc) y ablación de NAcc, de ocho pacientes con anorexia, donde los hallazgos sugieren que la operación mejoró la conducta alimentaria de las pacientes con $\mathrm{AN}$, lo que a su vez mejoró su condición física. Así mismo, los resultados indicaron que los síntomas de TOC, depresión y ansiedad disminuyeron después de la operación y que el nivel cognitivo preoperatorio mostró mejoría. Para el caso de TOCR y DR, Kubu et al.,(2013) presentaron datos de 21 pacientes (diez con TOC y once con DR) tratados a través de ECP [Estimulación cerebral profunda] de la cápsula ventral y el estriado ventral, reportando mejorías clínicas en los perfiles psiquiátrico, afectivo y neuropsicológico.

Actualmente, continúan teniendo gran eficacia los procedimientos ablativos (Wang, et al, 2013), destacándose dentro de la patología psiquiátrica la cingulotomía y la talamotomía. Por otro lado, también la termoablación de estas estructuras límbicas ha demostrado alteración en la respuesta autonómica. Un procedimiento reciente que se ha estudiado es la ECP (Luigjes, et al.,2013; Moreines, McClintock \& Holzheimer, 2011), estrategia cuya finalidad es estimular un blanco específico dentro del cerebro, y que se lleva a cabo para tratar determinadas patologías psiquiátricas, por ejemplo, en el núcleo accumbens. Se ha demostrado (Grubert, 2011) que este tipo de intervención interrumpe, inhibe y/o estimula el "circuito de recompensa" que se inicia en el lóbulo frontal y termina en el tegmentario mesencefálico, favoreciendo la inhibición de respuestas estereotipadas disfuncionales y/o disminuyendo los síntomas depresivos en trastornos obsesivos compulsivos y en depresión. La ECP implica la colocación de un electrodo por el cual pasa una corriente que inhibe o excita la función de las zonas o núcleos alterados. Estos electrodos funcionan como un marcapasos de la actividad nerviosa. Con cualquiera de estos procedimientos se consigue que cedan los síntomas excitadores o que se desactive la influencia inhibidora de determinados centros, balanceando de nuevo la actividad inhibidora-excitadora cerebral (Méndez, et al., 2009).

Son limitadas las investigaciones en las que se ha descrito el perfil psicológico, neuropsicológico y funcional de estos pacientes debido a que la visión de análisis predominante es de tipo médico-psiquiátrico (Wang, et al., 2013; Kubu, et al., 2013; McLaughlin, et al. 2013). Sin embargo, es de vital importancia tener un instrumento de medición objetiva del perfil funcional y del resto de los perfiles mencionados en los pacientes que son sometidos a este tipo de intervenciones.

Se considera importante incluir el estudio comparativo de la funcionalidad del paciente antes y después de la cirugía para conocer los efectos que tiene la misma en el desempeño de sus actividades de la vida diaria, las cuales se han visto severamente afectadas por la patología psiquiátrica.
Como se ha descrito previamente, esta afectación severa en el nivel de funcionamiento, junto con otras variables, es indispensable para considerar a la patología como resistente.

La amplia gama de trastornos psiquiátricos genera un impacto significativo en la funcionalidad de los pacientes, razón por la cual debe evaluarse la influencia de los procedimientos terapéuticos sobre el nivel de funcionamiento de pacientes con patología psiquiátrica resistente (Guridi \& Aldave, 2011). El objetivo de este estudio fue describir los cambios encontrados en el perfil funcional de 13 pacientes con Trastorno de la Conducta Alimentaria Resistente (TCAR), Trastorno Obsesivo Compulsivo y Depresión Resistentes (TOC y DR) o Depresión Resistente (DR), a quienes se les realizó un procedimiento neuroquirúrgico altamente especializado para la disminución de síntomas psiquiátricos graves y resistentes.

\section{MÉTODO}

\section{Participantes}

El presente estudio incluyó a 13 pacientes, siete de ellos diagnosticados con algún Trastorno de la Conducta Alimentaria Resistente (TCAR), tres diagnosticados con Trastorno Obsesivo Compulsivo y Depresión Resistentes (TOC y DR), y tres pacientes diagnosticados con DR. Estos pacientes fueron seleccionados por un equipo interdisciplinario como candidatos a NFE para reducción de síntomas psiquiátricos y se les realizaron procedimientos de cingulotomía y talamotomía bilaterales para TCAR, colocación de neuroestimulador bilateral en núcleo accumbens y termoablación bilateral de cíngulo para TOC y $\mathrm{DR}$, así como estimulación cerebral profunda del núcleo accumbens para DR, entre 2010 y 2012 (véase Tabla 1). Los pacientes fueron seleccionados en términos de cronicidad, refiriéndose al tiempo de desarrollo de la patología; discapacidad, en cuanto a la afectación de la patología en la vida cotidiana del paciente; gravedad, haciendo referencia a la intensidad de la misma; así como a la falta de respuesta (resistencia) a los tratamientos convencionales, principalmente farmacológicos, físicos (TEC) y/o psicoterapéuticos. Por lo anterior, los criterios de inclusión para los tres grupos diagnósticos fueron: diagnóstico de acuerdo al DSM-IVTR, edad mínima 18 años sin límite en la edad máxima, ambos géneros, evolución de la enfermedad de más de dos años (demostrado por historia clínica), la severidad de los síntomas debía tener más de dos años con por lo menos cuatro ensayos terapéuticos con supervisión de adhesión al tratamiento en los que se hubiera demostrado fracaso farmacológico y psicoterapéutico, presencia de auto y heteroagresividad con riesgo para la integridad física, incluyendo intentos suicidas, discapacidad documentada y evaluada con 
la Escala de Sheehan, y firma de consentimiento informado por un familiar responsable. Los criterios de exclusión fueron: alguna contraindicación médica que impidiera la cirugía, riesgo quirúrgico elevado mediante APACHE II, solicitud de retiro del estudio por parte del responsable legal del paciente, falta de participación en las evaluaciones preoperatorias, falta de participación y abandono de las valoraciones posteriores al procedimiento. Los criterios de inclusión y exclusión antes citados han sido reportados y pueden ser revisados en Hernández, Zárate, Valencia, Meneses, Ochoa, Torres, Martínez (2009) y en Hernández, Zárate, Valencia, Meneses, García, Luján, Juárez, Lucino y Martínez (2009). Todos los pacientes completaron las evaluaciones pre y post quirúrgicas, aceptando la cirugía como medio para tratar la sintomatología de la enfermedad; tanto ellos como sus familiares firmaron el consentimiento informado.

Tabla 1.

Datos demográficos de los participantes en el estudio y procedimiento quirúrgico realizado.

\begin{tabular}{|c|c|c|c|c|}
\hline Paciente & Diagnóstico & Edad & Sexo & $\begin{array}{c}\text { Procedimiento y blancos } \\
\text { quirúrgicos }\end{array}$ \\
\hline 1 & TACR & 24 & $F$ & $\begin{array}{l}\text { Cingulotomía y } \\
\text { Talamotomía bilaterales }\end{array}$ \\
\hline 2 & TCAR & 41 & $\mathrm{~F}$ & Talamotomia bilateral \\
\hline 3 & TCAR & 43 & $\mathrm{~F}$ & $\begin{array}{l}\text { Cingulotomía y } \\
\text { Talamotomía bilaterales }\end{array}$ \\
\hline 4 & TCAR & 20 & $\mathrm{~F}$ & $\begin{array}{l}\text { Cingulotomía y } \\
\text { Talamotomía bilaterales }\end{array}$ \\
\hline 5 & TCAR & 40 & $\mathrm{~F}$ & $\begin{array}{l}\text { Cingulotomía y } \\
\text { Talamotomía bilaterales }\end{array}$ \\
\hline 6 & TCAR & 36 & $\mathrm{~F}$ & $\begin{array}{l}\text { Cingulotomía y } \\
\text { Talamotomía bilaterales }\end{array}$ \\
\hline 7 & TCAR & 29 & $\mathrm{~F}$ & $\begin{array}{l}\text { Cingulotomía y } \\
\text { Talamotomía bilaterales }\end{array}$ \\
\hline 8 & TOC y DR & 51 & $\mathrm{~F}$ & $\begin{array}{l}\text { ECP del NAcc y } \\
\text { termoablación bilateral de } \\
\text { cíngulo }\end{array}$ \\
\hline 9 & TOC y DR & 46 & $\mathrm{~F}$ & $\begin{array}{l}\text { ECP del NAcc y } \\
\text { termoablación bilateral de } \\
\text { cíngulo }\end{array}$ \\
\hline 10 & TOC y DR & 35 & $\mathrm{~F}$ & $\begin{array}{l}\text { ECP del NAcc y } \\
\text { termoablación bilateral } \\
\text { de cíngulo }\end{array}$ \\
\hline 11 & DR & 47 & M & ECP del NAcc \\
\hline 12 & DR & 45 & $\mathrm{~F}$ & ECP del NAcc \\
\hline 13 & DR & 60 & $\mathrm{~F}$ & ECP del NAcc \\
\hline
\end{tabular}

TCAR: Trastorno de la Conducta Alimentaria Resistente; TOC: Trastorno Obsesivo Compulsivo; DR: Depresión Resistente; ECP: Estimulación Cerebral Profunda; NAcc: Núcleo Acumbens.

\section{Diseño experimental}

Todos los pacientes del estudio presentaban una condición de resistencia a tratamientos convencionales indicados, la cual fue documentada por un equipo interdisciplinario experto. En la investigación no se incluye un grupo control; en su lugar se realizó un diseño pre/post tratamiento.

\section{Instrumentos}

Se empleó el instrumento Eje V de Kennedy (Eje K) Evaluación psiquiátrica del funcionamiento del paciente (Kennedy, 2003), con el objetivo de evaluar el perfil funcional de los pacientes, el cual aporta datos que permiten valorar el funcionamiento global del paciente psiquiátrico en su vida cotidiana a través de la identificación de diversos síntomas, durante la semana anterior a la aplicación del mismo. El Eje K consta de siete subescalas (véase Tabla 2) y así mismo proporciona una Escala de Evaluación de la Actividad Global (EAG) que indica el nivel de funcionamiento general del individuo. Se puntúa de 0 a 100 en donde 100 representa el nivel máximo de funcionamiento.

\section{Procedimiento}

Se realizaron dos mediciones: La primera fue tomada una semana previa al tratamiento quirúrgico; los blancos quirúrgicos para TCAR fueron talamotomía ( $\mathrm{n}=1$ y cingulotomía $(n=6)$, en tanto que para TOC y TCAR $(n=3)$ fue colocado un neuroestimulador bilateral en el núcleo accumbens y se hizo termoablación bilateral de cíngulo; y para DR $(n=3)$ fue colocado un neuroestimulador bilateral en el núcleo accumbens (tabla 1). Los detalles del procedimiento quirúrgico pueden ser consultados en publicaciones previas (Hernández, Zárate, Valencia, Meneses, García, Luján, Juárez, Lucino \& Martínez, 2009). La segunda medición fue tomada seis meses posteriores a la citada intervención quirúrgica.

\section{Análisis de datos}

Se empleó la Prueba Pareada de Wilcoxon y la Prueba de Cohen para el grupo de pacientes con TCAR. Para el caso de TOCR/DR y DP se presentan las puntuaciones por caso.

\section{RESULTADOS}

Los resultados muestran una tendencia de cambios positivos entre las puntuaciones pre y postratamiento en los tres grupos de PPR, tanto en la EAG como en las subescalas que constituyen el instrumento; sin embargo, dado el tamaño limitado de la muestra se realizaron análisis con estadística no paramétrica para el grupo TCAR (véase tabla 2). Como puede observarse para este grupo, la EAG mostró cambios positivos globales pretratamiento $(M d=56)$ y postratamiento 
$(M d=84)$ siendo esta diferencia estadísticamente significativa $(\mathrm{z}=-2.36, \mathrm{p}<0.05)$ y con un tamaño del efecto grande $(\mathrm{d}=2.24)$. Dentro de las subescalas que presentaron mayor cambio positivo se observó el que ocurrió en habilidades ocupacionales entre el pre $(M d=50)$ y el postratamiento $(\mathrm{Md}=90)(\mathrm{z}=-2.41, \mathrm{p}<0.05)$. Cabe mencionar que mientras las puntuaciones se encuentren más cercanas a 100, el funcionamiento en la vida cotidiana es mejor. Los valores medios observados de la fase pre a la fase post en el grupo diagnóstico TCAR se resumen en la tabla 2.

Tabla 2.

Comparación de medianas a través de prueba pareada de Wilcoxon. Grupo diagnóstico TCAR

\begin{tabular}{lllll}
\hline \multicolumn{1}{c}{ Escalas } & Md Pre & \multicolumn{1}{c}{$\boldsymbol{M} \boldsymbol{d}$ Post } & $\mathbf{Z}$ & $\mathbf{P}$ \\
\hline D. Psicológico & 50 & 75 & -2.41 & .016 \\
Violencia & 60 & 90 & -2.38 & .017 \\
H. Sociales & 65 & 80 & -2.46 & .014 \\
H. Ocupacionales & 50 & 90 & -2.41 & .016 \\
Ab. Sustancias & 100 & 100 & -.577 & .564 \\
D. médico & 60 & 90 & -2.4 & .016 \\
D. Secundario & 70 & 90 & -2.23 & .026 \\
EAG & 56 & 84 & -2.37 & .018 \\
\hline
\end{tabular}

Nota. D. Deterioro; H. Habilidades, Ab. Abuso; EAG. Escala de Actividad General

\section{$T O C / D R$ y $D R$}

En todas las dimensiones que integran el perfil funcional (Eje K), se observaron cambios positivos entre los valores globales pretratamiento y postratamiento, tal como se muestra en las tablas 3 y 4 . Como puede observarse, el grupo de DR presentó cambios positivos mayores que los que se observaron en las puntuaciones del grupo TOC/DR.

Tabla 3.

Datos de la escala EJE K por paciente con TOCR/DR pre y post tratamiento

\begin{tabular}{lrlllll}
\hline \multirow{2}{*}{ Escalas } & \multicolumn{3}{c}{$\begin{array}{c}\text { Puntuaciones Pre } \\
\text { Tratamiento }\end{array}$} & \multicolumn{4}{c}{$\begin{array}{c}\text { Puntuaciones Post } \\
\text { Tratamiento }\end{array}$} \\
\cline { 2 - 7 } & \multicolumn{1}{c}{1} & \multicolumn{1}{c}{2} & \multicolumn{1}{c}{3} & 1 & 2 & \multicolumn{1}{c}{3} \\
\hline D. Psicológico & 50 & 50 & 50 & 80 & 60 & 60 \\
Violencia & 50 & 40 & 45 & 90 & 50 & 50 \\
H. Sociales & 70 & 50 & 70 & 80 & 60 & 80 \\
H. Ocupacionales & 50 & 50 & 50 & 80 & 60 & 70 \\
Ab. Sustancias & 100 & 70 & 100 & 100 & 75 & 100 \\
D. médico & 90 & 90 & 90 & 90 & 90 & 90 \\
D. Secundario & 90 & 90 & 90 & 90 & 90 & 90 \\
EAG & 68 & 48 & 54 & 83 & 58 & 65 \\
\hline
\end{tabular}

Nota. D. Deterioro; H. Habilidades, Ab. Abuso; EAG. Escala de Actividad General
Tabla 4.

Datos de la escala EJE K por paciente con DR pre y post tratamiento

\begin{tabular}{lrrrrrr}
\hline \multirow{1}{*}{ Escalas } & \multicolumn{2}{c}{$\begin{array}{c}\text { Puntuaciones Pre } \\
\text { Tratamiento }\end{array}$} & \multicolumn{3}{c}{$\begin{array}{c}\text { Puntuaciones } \\
\text { Post } \\
\text { Tratamiento }\end{array}$} \\
\cline { 2 - 7 } D. Psicológico & \multicolumn{1}{c}{1} & \multicolumn{1}{c}{2} & 3 & 1 & 2 & 3 \\
\cline { 2 - 7 } Violencia & 50 & 50 & 50 & 80 & 80 & 75 \\
H. Sociales & 90 & 45 & 40 & 90 & 80 & 75 \\
H. Ocupacionales & 50 & 60 & 65 & 80 & 70 & 75 \\
Ab. Sustancias & 100 & 100 & 100 & 100 & 100 & 100 \\
D. médico & 90 & 90 & 90 & 90 & 90 & 90 \\
D. Secundario & 90 & 90 & 90 & 90 & 90 & 90 \\
EAG & 54 & 51 & 51 & 80 & 78 & 76 \\
\hline
\end{tabular}

Nota. D. Deterioro; H. Habilidades, Ab. Abuso; EAG. Escala de Actividad General

\section{DISCUSIÓN}

Se realizó un estudio pre y postratamiento valorando el perfil funcional (a través de la escala EJE K) de pacientes sometidos a tratamiento de NFE para patología psiquiátrica resistente. Dichos pacientes padecían una enfermedad con síntomas graves, crónicos y resistentes, por lo que esta intervención terapéutica se presenta como una opción en los casos en donde los tratamientos convencionales no contribuyen de forma importante a la mejoría clínica del paciente.

La eficacia del tratamiento neuroquirúrgico propuesto ha sido evaluada mediante diferentes parámetros en diversos perfiles clínicos de análisis, tales como el cognitivo-intelectual, el neuropsicológico, el de personalidad, el psiquiátrico y el funcional; sin embargo, en este artículo se presentan únicamente los resultados obtenidos de la evaluación del perfil funcional por considerarse de especial relevancia para el paciente, por lo que el objetivo del presente trabajo fue valorar los cambios generados por el tratamiento de NFE en el perfil funcional a través del instrumento EJE K. Los resultados indican cambios positivos significativos para el grupo de TCAR en las escalas que componen dicho perfil así como en la EAG, en tanto que para los pacientes TOC/DR y DR se presentan las puntuaciones que muestran cambios positivos igualmente en todas las subescalas y en la EAG.

TCAR

Como se describió en la EAG, este grupo diagnóstico presentó cambios positivos, denotando mejoría clínica en las subescalas de habilidades ocupacionales, deterioro psicológico, deterioro médico y habilidades sociales, principalmente. Quedó demostrado que se alcanzó el 
propósito principal de la NFE, consistente en aliviar, tanto el sufrimiento psicológico, como aumentar el nivel de funcionamiento de los pacientes con estas características.

\section{$T O C / D R$}

Los datos indican que los cambios que presentaron estos pacientes, aunque positivos en todos las subescalas y en la EAG, son menos evidentes en comparación con los presentados por los grupos TCAR y DR, siendo éstos los más grandes y constantes en los tres pacientes en las subescalas de Deterioro Psicológico y Habilidades Ocupacionales, lo que al igual que en los citados grupos es un probable indicador de éxito del tratamiento

$D R$

$\mathrm{Al}$ aumentar probablemente la motivación y los comportamientos de búsqueda de recompensa mediante la ECP en Nacc se obtuvo una mejoría global cualitativa en el funcionamiento de este grupo de pacientes, así como en todas las subescalas.

En general, los resultados mostraron cambios de mejoría en el perfil de funcionamiento de todos los grupos diagnósticos y de todos los pacientes evaluados, siendo el grupo de los Trastornos de la Conducta Alimentaria Resistente el que presentó diferencias estadísticamente significativas por ser una muestra más grande. Las dimensiones que mostraron cambios con mayor evidencia en los tres grupos fueron en las subescalas Deterioro Psicológico y Habilidades Ocupacionales.

La investigación presentada sobre cambios en el perfil funcional en pacientes con PPR sometidos a NFE para la reducción de los síntomas psiquiátricos en distintos padecimientos, tiene como uno de sus objetivos académicos, mostrar también la importancia de la contribución de la psicología y la neuropsicología clínica en las intervenciones terapéuticas de alta complejidad como ocurrió en este caso (Mustaca, 2003). De acuerdo con Vilar (1997), este tipo de intervenciones tiene entre sus atributos el trabajo interdisciplinario, su carácter innovador y el empleo de los conocimientos, experiencias y tecnologías más avanzadas; todo ello, en el campo de las neurociencias del siglo XXI, contexto, en el que también se analizaron los resultados obtenidos y que fueron descritos previamente.

La NFE representa una opción terapéutica emergente efectiva orientada a disminuir el sufrimiento de los pacientes con patología psiquiátrica resistente, así como a mejorar su nivel de funcionamiento global y, por ende, su calidad de vida. Es por esto que resulta fundamental evaluar el perfil funcional de forma pre y posquirúrgica, entre otras variables psicológicas y neuropsicológicas de suma importancia.
Es importante mencionar, como limitaciones del estudio, y como es el caso de la mayoría de los estudios realizados en este campo, que el tamaño de las muestras es pequeño, debido a la alta especificidad de las características de los pacientes seleccionados para ser tratados mediante NFE, por lo que los resultados no pueden ser concluyentes. Sin embargo, quedan aún estudios por realizar que deberán incluir una mayor cohorte, otras variables psicológicas de análisis, grupos homogéneos de comparación entre procedimientos, el impacto clínico de procedimientos quirúrgicos combinados y un tiempo más prolongado de seguimiento.

\section{REFERENCIAS}

Carrillo, P., Jiménez, A., Soto, C., Velasco, F. \& Castro, R. (2006). Introducción a la neurocirugía psiquiátrica. Salud Mental, 29, 3-12.

Grubert, C., Hurlemann, R., Bwerenick, B.H., Kayser, S., Hadrysiewicz, B., Acmacher, N., Turm, V. \& Schlaepfer, T.E. (2011). Neuropsychological safety of nucleus accumbens. Deep brain stimulation for major depression: effects of 12month stimulation. World Journal Biology Psychiatric, 12, 516-527.

Guridi, J. \& Aldave G. (2011). Dianas quirúrgicas en el tratamiento de enfermedades psiquiátricas. Desde el movimiento a las emociones. Neurocirugía, 22, 5-22.

Hernández, S., Zárate, M., Valencia, G., Meneses, Ochoa, M.G., Torres, S., Martínez, B. (2009). El papel actual de la cirugía del sistema límbico: El caso de la anorexia y bulimia terminales. Archivos Neurociencias, 14, 11-21.

Hernández, S., Zárate, M., Valencia, G., Meneses, L., García, G., Luján, G., Juárez, C., Lucino, C. \& Martínez, S. (2009). Blancos estereotácticos más comunes en neurocirugía funcional, experiencia en el Centro Médico Nacional "20 de Noviembre". Archivos Neurociencias, 14, 94-101.

Kennedy, J.A. (2003). Mastering the Kennedy Axis V: A new Psychiatric Assessment of Patient Functioning (pp. 66-90). American Psychiatric Publications.

Kubu, C. S., Malone, D. A., Chelune, G., Malloy, P., Rezai, A. R., \& Greenberg, B. D. (2013). Neuropsychology Outcome after Deep Brain Stimulation in the Ventral Capsule/Ventral Striatum for Highly Refractory Obsessive-Compulsive Disorder of Major Depression. Stereotactic and Functional Neurosurgey, 91, 374-378.

Luigjes,J., Kwaasteniet B.P., De Koning P.P., Oudjin, M.S., Van de Munckhof, P., Schuurman, P.R. \& Denys, D. (2013). Surgey for Psychiatric Disorders. World Neurosurgery, 80, 17-31.

McLaughlin, N., Didie, E., Machado, A., Haber, S., Eskandar, E. \& Greenberg, B. (2013). Improvements in Anorexia Symptoms After Deep Brain Stimulation for Intractable Obsessive-Compulsive Disorder. Biological Psychiatry Journal, 72, 29-31. 
Méndez, F., Ochoa, M., Morel, T., Valencia, G., Hernández, S. \& Zárate, M. (2009). Cirugía del sistema límbico en pacientes con patología psiquiátrica severa refractaria: Revisión de casos. Revista Mexicana de Neurociencias, 10, 434-442.

Moreines, J.L., McClintock, S.M. \& Holzheimer, P.E. (2011). Neuropsychologic effets for neuromodulation techniques for treatment-resistant depression: A review. Brain Stimulation, 4, 17-27.

Mustaca, A.E. (2003). Análisis Experimental del Comportamiento y Neurociencias. Acta Colombiana de Psicología, 10, 7-23.

Oquendo, R.V. (2003). Depresión Refractaria: Actualización Conceptual y Clínica. Asociación Psiquiátrica de América Latina. 13, 41-56.
Thase, M.E. (2003). Effectiveness of Antidepressants: Comparative Remission Rates. Journal of Clinical Psychiatry, 64, 3-7.

Vilar, S. (1997). La nueva racionalidad: comprender la complejidad con métodos transdisciplinarios (pp. 120-163). Barcelona: Kairós.

Wang, J., Chang, C., Geng, N., Wang, X. \& Gao, G. (2013). Treatment of Intractable Anorexia Nervosa with Inactivation of the Nucleus Accumbens Using Stereotactic Surgey. Stereotactic and Functional Neurosurgey, 91, 364-372. 